\title{
A parametric family of quartic Thue equations
}

\author{
by \\ Andrej Dujella (Zagreb) and Borka JadriJević (Split)
}

1. Introduction. In 1909, Thue [23] proved that an equation $F(x, y)=$ $m$, where $F \in \mathbb{Z}[X, Y]$ is a homogeneous irreducible polynomial of degree $n \geq 3$ and $m \neq 0$ a fixed integer, has only finitely many solutions. His proof was not effective. In 1968, Baker [2] gave an effective bound based on the theory of linear forms in logarithms of algebraic numbers. In recent years general powerful methods have been developed for the explicit solution of Thue equations (see $[19,26,6]$ ), following from Baker's work.

Thomas [22] was the first to investigate a parametrized family of Thue equations. Since then, several families have been studied (see [12] for references). In particular, quartic families have been considered in $[7,12,13,15$, $18,20,24,27,28]$.

In this paper, we consider the equation

$$
x^{4}-4 c x^{3} y+(6 c+2) x^{2} y^{2}+4 c x y^{3}+y^{4}=1,
$$

and we prove that for $c \geq 3$ it has no solution except the trivial ones: $( \pm 1,0),(0, \pm 1)$.

We will apply the method of Tzanakis. In [25], Tzanakis considered the equations of the form $f(x, y)=m$, where $f$ is a quartic form whose corresponding quartic field $\mathbf{K}$ is Galois and non-cyclic. By [17], this condition on $\mathbf{K}$ is equivalent to $\mathbf{K}$ having three quadratic subfields, which happens exactly when the cubic resolvent of the quartic Thue equation has three distinct rational roots. Assuming that $\mathbf{K}$ is not totally complex, we conclude that $\mathbf{K}$ is totally real, in fact, it is a compositum of two real quadratic fields and it contains exactly three quadratic subfields, all of which are real. Tzanakis showed that solving the equation $f(x, y)=m$, under the above assumptions on $\mathbf{K}$, reduces to solving a system of Pellian equations.

2000 Mathematics Subject Classification: Primary 11D25, 11D59; Secondary 11B37, 11J68, 11J86, $11 \mathrm{Y} 50$.

Key words and phrases: Thue equations, simultaneous Pellian equations, linear forms in logarithms. 
We will show that solving (1) by the method of Tzanakis reduces to solving the system

$$
\begin{aligned}
(2 c+1) U^{2}-2 c V^{2} & =1, \\
(c-2) U^{2}-c Z^{2} & =-2 .
\end{aligned}
$$

We will find a lower bound for solutions of this system using the "congruence method" introduced in [11] and used also in $[9,10]$. The comparison of this lower bound with an upper bound obtained from a theorem of Bennett [5] on simultaneous approximations of algebraic numbers finishes the proof for $c \geq 179559$. For $c \leq 179558$ we use a theorem a Baker and Wüstholz [4] and a version of the reduction procedure due to Baker and Davenport [3].

There are three reasons why we have chosen the family (1). First of all, for all members of this family $(c \neq 0,1,2)$ the corresponding quartic field satisfies the above conditions, so the method of Tzanakis can be applied. Furthermore, the system of Pellian equations obtained in this way is very suitable for the application of both "congruence method" and Bennett's theorem.

Our main result is the following theorem.

Theorem 1. Let $c \geq 3$ be an integer. The only solutions to (1) are $(x, y)=( \pm 1,0)$ and $(0, \pm 1)$.

Let us note that the statement of Theorem 1 is trivially true for $c=0$ and $c=1$. On the other hand, for $c=2$ we have

$$
x^{4}-8 x^{3} y+14 x^{2} y^{2}+8 x y^{3}+y^{4}=\left(x^{2}-4 x y-y^{2}\right)^{2}=1,
$$

and therefore in this case our equation has infinitely many solutions given by $x=\frac{1}{2} F_{3 n+3}, y=\frac{1}{2} F_{3 n}$.

For $c=4$ we have

$$
x^{4}-16 x^{3} y+26 x^{2} y^{2}+16 x y^{3}+y^{4}=\left(x^{2}-8 x y-y^{2}\right)^{2}-(6 x y)^{2}=1,
$$

which clearly implies $x y=0$. Therefore we may assume that $c \neq 4$.

2. The method of Tzanakis. In this section we will describe the method of Tzanakis for solving quartic Thue equations whose corresponding quartic field $\mathbf{K}$ has the properties described in Section 1.

Consider the quartic Thue equation

$$
f(x, y)=m
$$

$$
f(x, y)=a_{0} x^{4}+4 a_{1} x^{3} y+6 a_{2} x^{2} y^{2}+4 a_{3} x y^{3}+a_{4} y^{4} \in \mathbb{Z}[x, y], \quad a_{0}>0 .
$$

We assign to this equation the cubic equation

$$
4 \varrho^{3}-g_{2} \varrho-g_{3}=0
$$


with roots opposite to those of the cubic resolvent of the quartic equation $f(x, 1)=0$. Here $g_{2}=a_{0} a_{4}-4 a_{1} a_{3}+3 a_{2}^{2} \in \frac{1}{12} \mathbb{Z}$,

$$
g_{3}=\left|\begin{array}{lll}
a_{0} & a_{1} & a_{2} \\
a_{1} & a_{2} & a_{3} \\
a_{2} & a_{3} & a_{4}
\end{array}\right| \in \frac{1}{432} \mathbb{Z} .
$$

By [25], the conditions on $\mathbf{K}$ from Section 1 are equivalent to the fact that the cubic equation (3) has three rational roots $\varrho_{1}, \varrho_{2}, \varrho_{3}$ and

$$
\frac{a_{1}^{2}}{a_{0}}-a_{2} \geq \max \left\{\varrho_{1}, \varrho_{2}, \varrho_{3}\right\} .
$$

Let $H(x, y)$ and $G(x, y)$ be the quartic and sextic covariants of $f(x, y)$, respectively (see [16, Chapter 25]), i.e.

$$
\begin{aligned}
& H(x, y)=-\frac{1}{144}\left|\begin{array}{cc}
\frac{\partial^{2} f}{\partial x^{2}} & \frac{\partial^{2} f}{\partial x \partial y} \\
\frac{\partial^{2} f}{\partial y \partial x} & \frac{\partial^{2} f}{\partial y^{2}}
\end{array}\right| \in \frac{1}{48} \mathbb{Z}[x, y], \\
& G(x, y)=-\frac{1}{8}\left|\begin{array}{ll}
\frac{\partial f}{\partial x} & \frac{\partial f}{\partial y} \\
\frac{\partial H}{\partial x} & \frac{\partial H}{\partial y}
\end{array}\right| \in \frac{1}{96} \mathbb{Z}[x, y] .
\end{aligned}
$$

Then $4 H^{3}-g_{2} H f^{2}-g_{3} f^{3}=G^{2}$. If we put $H=\frac{1}{48} H_{0}, G=\frac{1}{96} G_{0}, \varrho_{i}=\frac{1}{12} r_{i}$, $i=1,2,3$, then $H_{0}, G_{0} \in \mathbb{Z}[x, y], r_{i} \in \mathbb{Z}, i=1,2,3$, and

$$
\left(H_{0}-4 r_{1} f\right)\left(H_{0}-4 r_{2} f\right)\left(H_{0}-4 r_{3} f\right)=3 G_{0}^{2} \text {. }
$$

There exist positive square-free integers $k_{1}, k_{2}, k_{3}$ and quadratic forms $G_{1}, G_{2}, G_{3} \in \mathbb{Z}[x, y]$ such that

$$
H_{0}-4 r_{i} f=k_{i} G_{i}^{2}, \quad i=1,2,3,
$$

and $k_{1} k_{2} k_{3}\left(G_{1} G_{2} G_{3}\right)^{2}=3 G_{0}^{2}$. If $(x, y) \in \mathbb{Z} \times \mathbb{Z}$ is a solution of (2), then

$$
\begin{aligned}
& k_{2} G_{2}^{2}-k_{1} G_{1}^{2}=4\left(r_{1}-r_{2}\right) m, \\
& k_{3} G_{3}^{2}-k_{1} G_{1}^{2}=4\left(r_{1}-r_{3}\right) m .
\end{aligned}
$$

In this way, solving the Thue equation (2) reduces to solving the system of Pellian equations (5) and (6) with one common unknown.

3. The system of Pellian equations. Let us apply the method from Section 2 to the equation

$$
f(x, y)=x^{4}-4 c x^{3} y+(6 c+2) x^{2} y^{2}+4 c x y^{3}+y^{4}=1 .
$$


We have

$$
\begin{gathered}
g_{2}=\frac{1}{3}\left(21 c^{2}+6 c+4\right), \\
g_{3}=-\frac{1}{27}\left(81 c^{3}+99 c^{2}-18 c-8\right), \\
\varrho_{1}=\frac{1}{2} c+\frac{2}{3}, \quad \varrho_{2}=c-\frac{1}{3}, \quad \varrho_{3}=-\frac{3}{2} c-\frac{1}{3} .
\end{gathered}
$$

The condition (4) is clearly satisfied.

Furthermore, we obtain

$$
\begin{aligned}
& H_{0}-4 r_{1} f=24(c-2)(2 c+1)\left(x^{2}+y^{2}\right)^{2}, \\
& H_{0}-4 r_{2} f=48 c(c-2)\left(x^{2}+x y-y^{2}\right)^{2}, \\
& H_{0}-4 r_{3} f=24 c(2 c+1)\left(-x^{2}+4 x y+y^{2}\right)^{2} .
\end{aligned}
$$

Hence we may take

$$
\begin{aligned}
& k_{1}=6(c-2)(2 c+1), \quad k_{2}=3 c(c-2), \quad k_{3}=6 c(2 c+1), \\
G_{1}= & 2\left(x^{2}+y^{2}\right), \quad G_{2}=4\left(x^{2}+x y-y^{2}\right), \quad G_{3}=2\left(-x^{2}+4 x y+y^{2}\right) .
\end{aligned}
$$

Inserting this in (5) and (6) we obtain

$$
\begin{gathered}
c G_{2}^{2}-(4 c+2) G_{1}^{2}=-8 \\
c G_{3}^{2}-(c-2) G_{1}^{2}=8 .
\end{gathered}
$$

Let

$$
U=G_{1} / 2=x^{2}+y^{2}, \quad V=G_{2} / 4=x^{2}+x y-y^{2}, \quad Z=G_{3} / 2=-x^{2}+4 x y+y^{2} .
$$

Then from (7) and (8) we obtain the system of Pellian equations

$$
\begin{aligned}
(2 c+1) U^{2}-2 c V^{2} & =1, \\
(c-2) U^{2}-c Z^{2} & =-2 .
\end{aligned}
$$

LEMMA 1. Let $k \geq 2$ be an integer. If $x$ and $y$ are positive integers satisfying the Pellian equation

$$
(k-1) y^{2}-(k+1) x^{2}=-2,
$$

then there exists an integer $m \geq 0$ such that $x=x_{m}$ and $y=y_{m}$, where the sequences $\left(x_{m}\right)$ and $\left(y_{m}\right)$ are given by

$$
\begin{array}{lll}
x_{0}=1, & x_{1}=2 k-1, \quad x_{m+2}=2 k x_{m+1}-x_{m}, & m \geq 0 ; \\
y_{0}=1, & y_{1}=2 k+1, \quad y_{m+2}=2 k y_{m+1}-y_{m}, & m \geq 0 .
\end{array}
$$

Proof. See [8, p. 312].

Lemma 1 immediately yields

LEMMA 2. Let $(U, V, Z)$ be a positive integer solution of the system of Pellian equations (9) and (10). Then there exist nonnegative integers $m$ and $n$ such that

$$
U=v_{m}=w_{n},
$$


where the sequences $\left(v_{m}\right)$ and $\left(w_{n}\right)$ are given by

$$
\begin{array}{ccc}
v_{0}=1, & v_{1}=8 c+1, \quad v_{m+2}=(8 c+2) v_{m+1}-v_{m}, \quad m \geq 0, \\
w_{0}=1, \quad w_{1}=2 c-1, & w_{n+2}=(2 c-2) w_{n+1}-w_{n}, \quad n \geq 0 .
\end{array}
$$

Therefore, in order to prove Theorem 1, it suffices to show that $v_{m}=w_{n}$ implies $m=n=0$.

Solving recurrences (11) and (12) we find

$$
\begin{aligned}
v_{m}= & \frac{1}{2 \sqrt{4 c+2}}\left[(2 \sqrt{c}+\sqrt{4 c+2})(4 c+1+2 \sqrt{2 c(2 c+1)})^{m}\right. \\
& \left.-(2 \sqrt{c}-\sqrt{4 c+2})(4 c+1-2 \sqrt{2 c(2 c+1)})^{m}\right], \\
w_{n}= & \frac{1}{2 \sqrt{c-2}}\left[(\sqrt{c}+\sqrt{c-2})(c-1+\sqrt{c(c-2)})^{n}\right. \\
& \left.-(\sqrt{c}-\sqrt{c-2})(c-1-\sqrt{c(c-2)})^{n}\right] .
\end{aligned}
$$

\section{Congruence relations}

Lemma 3. Let the sequences $\left(v_{m}\right)$ and $\left(w_{n}\right)$ be defined by (11) and (12). Then for all $m, n \geq 0$ we have

$$
\begin{aligned}
& v_{m} \equiv 4 m(m+1) c+1\left(\bmod 64 c^{2}\right), \\
& w_{n} \equiv(-1)^{n-1}[n(n+1) c-1]\left(\bmod 4 c^{2}\right) .
\end{aligned}
$$

Proof. Both relations are obviously true for $m, n \in\{0,1\}$.

Assume that (15) is valid for $m-2$ and $m-1$. Then

$$
\begin{aligned}
v_{m} & =(8 c+2) v_{m-1}-v_{m-2} \\
& \equiv(8 c+2)[4 m(m-1) c+1]-[4(m-1)(m-2) c+1] \\
& \equiv c[8+8 m(m-1)-4(m-1)(m-2)]+1 \\
& =4 m(m+1) c+1\left(\bmod 64 c^{2}\right) .
\end{aligned}
$$

Assume that (16) is valid for $n-2$ and $n-1$. Then

$$
\begin{aligned}
w_{n} & =(2 c-2) w_{n-1}-w_{n-2} \\
& \equiv(2 c-2)(-1)^{n}[n(n-1) c-1]-(-1)^{n-1}[(n-1)(n-2) c-1] \\
& \equiv c(-1)^{n-1}[2+2 n(n-1)-(n-1)(n-2)]+(-1)^{n} \\
& =(-1)^{n-1}[n(n+1) c-1]\left(\bmod 4 c^{2}\right) .
\end{aligned}
$$

Suppose that $m$ and $n$ are positive integers such that $v_{m}=w_{n}$. Then, of course, $v_{m} \equiv w_{n}\left(\bmod 4 c^{2}\right)$. By Lemma 3 , we have $(-1)^{n} \equiv 1(\bmod 2 c)$ and therefore $n$ is even. 
Assume that $n(n+1)<\frac{4}{5} c$. Since $m \leq n$ we also have $m(m+1)<\frac{4}{5} c$. Furthermore, Lemma 3 implies

$$
4 m(m+1) c+1 \equiv 1-n(n+1) c\left(\bmod 4 c^{2}\right)
$$

and

$$
2 m(m+1) \equiv-\frac{n(n+1)}{2}(\bmod 2 c)
$$

Consider the positive integer

$$
A=2 m(m+1)+\frac{n(n+1)}{2} .
$$

We have $0<A<2 c$ and, by (17), $A \equiv 0(\bmod 2 c)$, a contradiction.

Hence $n(n+1) \geq \frac{4}{5} c$, which implies $n>\sqrt{0.8 c}-0.5$. Therefore we proved

Proposition 1. If $v_{m}=w_{n}$ and $m \neq 0$, then $n>\sqrt{0.8 c}-0.5$.

5. An application of a theorem of Bennett. It is clear that the solutions of the system (9) and (10) induce good rational approximations to the numbers

$$
\theta_{1}=\sqrt{\frac{2 c+1}{2 c}} \text { and } \theta_{2}=\sqrt{\frac{c-2}{c}} .
$$

More precisely, we have

Lemma 4. All positive integer solutions $(U, V, Z)$ of the system of Pellian equations (9) and (10) satisfy

$$
\left|\theta_{1}-\frac{V}{U}\right|<\frac{1}{4 c} \cdot U^{-2}, \quad\left|\theta_{2}-\frac{Z}{U}\right|<\frac{1}{\sqrt{c(c-2)}} \cdot U^{-2} .
$$

Proof. We have

$$
\begin{aligned}
\left|\theta_{1}-\frac{V}{U}\right| & =\left|\sqrt{\frac{2 c+1}{2 c}}-\frac{V}{U}\right|=\left|\frac{2 c+1}{2 c}-\frac{V^{2}}{U^{2}}\right| \cdot\left|\sqrt{\frac{2 c+1}{2 c}}+\frac{V}{U}\right|^{-1} \\
& <\frac{1}{2 c U^{2}} \cdot \frac{1}{2}=\frac{1}{4 c} \cdot U^{-2}
\end{aligned}
$$

and

$$
\begin{aligned}
\left|\theta_{2}-\frac{Z}{U}\right| & =\left|\sqrt{\frac{c-2}{c}}-\frac{Z}{U}\right|=\left|\frac{c-2}{c}-\frac{Z^{2}}{U^{2}}\right| \cdot\left|\sqrt{\frac{c-2}{c}}+\frac{Z}{U}\right|^{-1} \\
& <\frac{2}{c U^{2}} \cdot \frac{1}{2} \sqrt{\frac{c}{c-2}}=\frac{1}{\sqrt{c(c-2)}} \cdot U^{-2} . \text { - }
\end{aligned}
$$

The numbers $\theta_{1}$ and $\theta_{2}$ are square roots of rationals which are very close to 1. For simultaneous Diophantine approximations to such kind of numbers there are very useful effective results of Masser and Rickert [14] and Bennett [5]. Let us mention that the first effective results on simultaneous 
approximation to fractional powers of rationals close to 1 were given by Baker in [1]. We will use the following theorem of Bennett [5, Theorem 3.2].

TheOREM 2. If $a_{i}, p_{i}, q$ and $N$ are integers for $0 \leq i \leq 2$, with $a_{0}<$ $a_{1}<a_{2}, a_{j}=0$ for some $0 \leq j \leq 2, q$ nonzero and $N>M^{9}$, where

$$
M=\max _{0 \leq i \leq 2}\left\{\left|a_{i}\right|\right\}
$$

then

$$
\max _{0 \leq i \leq 2}\left\{\left|\sqrt{1+\frac{a_{i}}{N}}-\frac{p_{i}}{q}\right|\right\}>(130 N \gamma)^{-1} q^{-\lambda}
$$

where

$$
\lambda=1+\frac{\log (33 N \gamma)}{\log \left(1.7 N^{2} \prod_{0 \leq i<j \leq 2}\left(a_{i}-a_{j}\right)^{-2}\right)}
$$

and

$$
\gamma= \begin{cases}\frac{\left(a_{2}-a_{0}\right)^{2}\left(a_{2}-a_{1}\right)^{2}}{2 a_{2}-a_{0}-a_{1}} & \text { if } a_{2}-a_{1} \geq a_{1}-a_{0}, \\ \frac{\left(a_{2}-a_{0}\right)^{2}\left(a_{1}-a_{0}\right)^{2}}{a_{1}+a_{2}-2 a_{0}} & \text { if } a_{2}-a_{1}<a_{1}-a_{0} .\end{cases}
$$

We will apply Theorem 2 with $a_{0}=-4, a_{1}=0, a_{2}=1, N=2 c, M=4$, $q=U, p_{0}=Z, p_{1}=U, p_{2}=V$. If $c \geq 131073$, then the condition $N>M^{9}$ is satisfied and we obtain

$$
\left(130 \cdot 2 c \cdot \frac{400}{9}\right)^{-1} U^{-\lambda}<\frac{1}{\sqrt{c(c-2)}} \cdot U^{-2} .
$$

If $c \geq 172550$ then $2-\lambda>0$ and (18) implies

$$
\log U<\frac{9.355}{2-\lambda}
$$

Furthermore,

$$
\frac{1}{2-\lambda}=\frac{1}{1-\frac{\log \left(\frac{26400}{9} c\right)}{\log \left(0.017 c^{2}\right)}}<\frac{\log \left(0.017 c^{2}\right)}{\log (0.00000579 c)} .
$$

On the other hand, from (14) we find that

$$
w_{n}>(c-1+\sqrt{c(c-2)})^{n}>(2 c-3)^{n},
$$

and Proposition 1 implies that if $(m, n) \neq(0,0)$, then

$$
U>(2 c-3)^{\sqrt{0.8 c}-0.5} \text {. }
$$

Therefore,

$$
\log U>(\sqrt{0.8 c}-0.5) \log (2 c-3) .
$$

Combining (19) and (20) we obtain 


$$
\sqrt{0.8 c}-0.5<\frac{9.355 \log \left(0.017 c^{2}\right)}{\log (2 c-3) \log (0.00000579 c)}
$$

and (21) leads to a contradiction if $c \geq 179559$. Therefore we proved

Proposition 2. If $c$ is an integer such that $c \geq 179559$, then the only solution of the equation $v_{m}=w_{n}$ is $(m, n)=(0,0)$.

6. The Baker-Davenport method. In this section we will apply the so-called Baker-Davenport reduction method in order to prove Theorem 1 for $3 \leq c \leq 179558$.

LeMma 5. If $v_{m}=w_{n}$ and $m \neq 0$, then

$$
\begin{aligned}
0< & n \log (c-1+\sqrt{c(c-2)})-m \log (4 c+1+2 \sqrt{2 c(2 c+1)}) \\
& +\log \frac{\sqrt{4 c+2}(\sqrt{c}+\sqrt{c-2})}{\sqrt{c-2}(2 \sqrt{c}+\sqrt{4 c+2})} \\
< & 0.627(4 c+1+2 \sqrt{2 c(2 c+1)})^{-2 m} .
\end{aligned}
$$

Proof. Define

$$
\begin{aligned}
& P=\frac{2 \sqrt{c}+\sqrt{4 c+2}}{\sqrt{4 c+2}}(4 c+1+2 \sqrt{2 c(2 c+1)})^{m}, \\
& Q=\frac{\sqrt{c}+\sqrt{c-2}}{\sqrt{c-2}}(c-1+\sqrt{c(c-2)})^{n} .
\end{aligned}
$$

From (13) and (14) it follows that the relation $v_{m}=w_{n}$ implies

$$
P+\frac{1}{2 c+1} P^{-1}=Q-\frac{2}{c-2} Q^{-1} .
$$

It is clear that $Q>P$. Furthermore,

$$
\frac{Q-P}{Q}=\frac{1}{Q}\left(\frac{1}{2 c+1} P^{-1}+\frac{2}{c-2} Q^{-1}\right)<P^{-2}\left(\frac{1}{2 c+1}+\frac{2}{c-2}\right) \leq \frac{15}{7} P^{-2} .
$$

Since $m, n \geq 1$, we have $P>8 c+1 \geq 25$ and $(Q-P) / Q<1 / 291$. Thus we may apply [21, Lemma B.2] to obtain

$$
\begin{aligned}
0 & <\log \frac{Q}{P}=-\log \left(1-\frac{Q-P}{Q}\right)<1.002 \cdot \frac{15}{7} P^{-2} \\
& =2.148 \cdot \frac{4 c+2}{(2 \sqrt{c}+\sqrt{4 c+2})^{2}}(4 c+1+2 \sqrt{2 c(2 c+1)})^{-2 m} \\
& <\frac{4.296(2 c+1)}{16 c}(4 c+1+2 \sqrt{2 c(2 c+1)})^{-2 m} \\
& <0.627(4 c+1+2 \sqrt{2 c(2 c+1)})^{-2 m} .
\end{aligned}
$$


Now we will apply the following famous theorem of Baker and Wüstholz [4]:

TheOREM 3. For a linear form $\Lambda \neq 0$ in logarithms of $l$ algebraic numbers $\alpha_{1}, \ldots, \alpha_{l}$ with rational integer coefficients $b_{1}, \ldots, b_{l}$ we have

$$
\log \Lambda \geq-18(l+1) ! l^{l+1}(32 d)^{l+2} h^{\prime}\left(\alpha_{1}\right) \ldots h^{\prime}\left(\alpha_{l}\right) \log (2 l d) \log B,
$$

where $B=\max \left\{\left|b_{1}\right|, \ldots,\left|b_{l}\right|\right\}$ and $d$ is the degree of the number field generated by $\alpha_{1}, \ldots, \alpha_{l}$.

Here

$$
h^{\prime}(\alpha)=\frac{1}{d} \max \{h(\alpha),|\log \alpha|, 1\},
$$

and $h(\alpha)$ denotes the standard logarithmic Weil height of $\alpha$.

We will apply Theorem 3 to the form from Lemma 5 . We have $l=3$, $d=4, B=n$,

$$
\begin{gathered}
\alpha_{1}=c-1+\sqrt{c(c-2)}, \quad \alpha_{2}=4 c+1+2 \sqrt{2 c(2 c+1)}, \\
\alpha_{3}=\frac{\sqrt{4 c+2}(\sqrt{c}+\sqrt{c-2})}{\sqrt{c-2}(2 \sqrt{c}+\sqrt{4 c+2})} .
\end{gathered}
$$

Under the assumption that $3 \leq c \leq 179558$ we find that

$$
h^{\prime}\left(\alpha_{1}\right)=\frac{1}{2} \log \alpha_{1}<\frac{1}{2} \log 2 c, \quad h^{\prime}\left(\alpha_{2}\right)=\frac{1}{2} \log \alpha_{2}<7.0889 .
$$

Furthermore, $\alpha_{3}<1.419$, and the conjugates of $\alpha_{3}$ satisfy

$$
\begin{aligned}
\left|\alpha_{3}^{\prime}\right| & =\frac{\sqrt{4 c+2}(\sqrt{c}-\sqrt{c-2})}{\sqrt{c-2}(2 \sqrt{c}+\sqrt{4 c+2})}<1 \\
\left|\alpha_{3}^{\prime \prime}\right| & =\frac{\sqrt{4 c+2}(2 \sqrt{c}+\sqrt{4 c+2})}{\sqrt{c-2}(\sqrt{c}+\sqrt{c-2})}<9.869 \\
\left|\alpha_{3}^{\prime \prime \prime}\right| & =\frac{\sqrt{4 c+2}(\sqrt{c}+\sqrt{c-2})(2 \sqrt{c}+\sqrt{4 c+2})}{2 \sqrt{c-2}}<1436471.1 .
\end{aligned}
$$

Therefore,

$$
h^{\prime}\left(\alpha_{3}\right)<\frac{1}{4} \log \left[(c-2)^{2} \cdot 1.419 \cdot 9.869 \cdot 1436471.1\right]<10.254 .
$$

Finally,

$$
\log \left[0.627(4 c+1+2 \sqrt{2 c(2 c+1)})^{-2 m}\right]<-2 m \log (8 c)<-2 m \log (2 c) .
$$

Hence, Theorem 3 implies

$$
2 m \log (2 c)<3.822 \cdot 10^{15} \cdot \frac{1}{2} \log (2 c) \cdot 7.0889 \cdot 10.254 \cdot \log n
$$

and

$$
m / \log n<6.946 \cdot 10^{16}
$$


By Lemma 5, we have

$$
\begin{aligned}
n \log (c-1+\sqrt{c(c-2)}) & <m \log (4 c+1+2 \sqrt{2 c(2 c+1)})+0.000931 \\
& <m \log [(4 c+1+2 \sqrt{2 c(2 c+1)}) \cdot 1.000932]
\end{aligned}
$$

and

$$
n / m<2.474 .
$$

Combining (22) and (23), we obtain

$$
n / \log n<1.719 \cdot 10^{17},
$$

which implies $n<7.471 \cdot 10^{18}$.

We may reduce this large upper bound using a variant of the BakerDavenport reduction procedure [3]. The following lemma is a slight modification of [11, Lemma 5a)]:

Lemma 6. Assume that $M$ is a positive integer. Let $p / q$ be a convergent of the continued fraction expansion of $\kappa$ such that $q>10 M$ and let $\varepsilon=$ $\|\mu q\|-M \cdot\|\kappa q\|$, where $\|\cdot\|$ denotes the distance from the nearest integer. If $\varepsilon>0$, then there is no solution of the inequality

$$
0<n-m \kappa+\mu<A B^{-m}
$$

in integers $m$ and $n$ with

$$
\frac{\log (A q / \varepsilon)}{\log B} \leq m \leq M .
$$

We apply Lemma 6 with

$$
\begin{gathered}
\kappa=\frac{\log \alpha_{2}}{\log \alpha_{1}}, \quad \mu=\frac{\log \alpha_{3}}{\log \alpha_{1}}, \quad A=\frac{0.627}{\log \alpha_{1}}, \\
B=(4 c+1+2 \sqrt{2 c(2 c+1)})^{2} \text { and } \quad M=7.471 \cdot 10^{18} .
\end{gathered}
$$

If the first convergent such that $q>10 M$ does not satisfy the condition $\varepsilon>0$, then we use the next convergent.

We performed the reduction from Lemma 6 for $3 \leq c \leq 179558, c \neq 4$. The use of the second convergent was necessary in 6810 cases $(3.79 \%)$, the third convergent was used in 143 cases $(0.08 \%)$, the fourth in 22 cases and the fifth in seven cases $(c=21027,22393,41842,56576,75541,96007$, 157920). In all cases we obtained $m \leq 7$. More precisely, we obtained $m \leq 7$ for $c=3 ; m \leq 6$ for $c \geq 5 ; m \leq 5$ for $c \geq 6 ; m \leq 4$ for $c \geq 13 ; m \leq 3$ for $c \geq 67 ; m \leq 2$ for $c \geq 724$. According to Proposition 1, this finishes the proof for $c \geq 79$. It is trivial to check that for $c \leq 78$ there is no solution of the equation $v_{m}=w_{n}$ with $(m, n) \neq(0,0)$ in the above ranges. 
Therefore, we have proved

Proposition 3. If $c$ is an integer such that $3 \leq c \leq 179558$, then the only solution of the equation $v_{m}=w_{n}$ is $(m, n)=(0,0)$.

Proof of Theorem 1. The statement follows directly from Propositions 2 and 3 .

Acknowledgements. The authors would like to thank Professor Attila Pethö for helpful suggestions on improvements of the first version of the manuscript.

\section{References}

[1] A. Baker, Simultaneous rational approximations to certain algebraic numbers, Proc. Cambridge Philos. Soc. 63 (1967), 693-702.

[2] - Contributions to the theory of Diophantine equations I. On the representation of integers by binary forms, Philos. Trans. Roy. Soc. London Ser. A 263 (1968), $173-191$.

[3] A. Baker and H. Davenport, The equations $3 x^{2}-2=y^{2}$ and $8 x^{2}-7=z^{2}$, Quart. J. Math. Oxford Ser. (2) 20 (1969), 129-137.

[4] A. Baker and G. Wüstholz, Logarithmic forms and group varieties, J. Reine Angew. Math. 442 (1993), 19-62.

[5] M. A. Bennett, On the number of solutions of simultaneous Pell equations, ibid. 498 (1998), 173-199.

[6] Yu. Bilu and G. Hanrot, Solving Thue equations of high degree, J. Number Theory 60 (1996), 373-392.

[7] J. H. Chen and P. M. Voutier, Complete solution of the Diophantine equation $X^{2}+$ $1=d Y^{4}$ and a related family of Thue equations, ibid. 62 (1996), 273-292.

[8] A. Dujella, The problem of the extension of a parametric family of Diophantine triples, Publ. Math. Debrecen 51 (1997), 311-322.

[9] —, Complete solution of a family of simultaneous Pellian equations, Acta Math. Inform. Univ. Ostraviensis 6 (1998), 59-67.

[10] —, An absolute bound for the size of Diophantine m-tuples, J. Number Theory 89 (2001), 126-150.

[11] A. Dujella and A. Pethö, Generalization of a theorem of Baker and Davenport, Quart. J. Math. Oxford Ser. (2) 49 (1998), 291-306.

[12] C. Heuberger, A. Pethő and R. F. Tichy, Complete solution of parametrized Thue equations, Acta Math. Inform. Univ. Ostraviensis 6 (1998), 93-113.

[13] G. Lettl and A. Pethö, Complete solution of a family of quartic Thue equations, Abh. Math. Sem. Univ. Hamburg 65 (1995), 365-383.

[14] D. W. Masser and J. H. Rickert, Simultaneous Pell equations, J. Number Theory 61 (1996), 52-66.

[15] M. Mignotte, A. Pethö and R. Roth, Complete solutions of quartic Thue and index form equations, Math. Comp. 65 (1996), 341-354.

[16] L. J. Mordell, Diophantine Equations, Academic Press, London, 1969.

[17] T. Nagell, Sur quelques questions dans la théorie des corps biquadratiques, Ark. Mat. 4 (1961), 347-376.

[18] A. Pethő, Complete solutions to families of quartic Thue equations, Math. Comp. 57 (1991), 777-798. 
[19] A. Pethő and R. Schulenberg, Effektives Lösen von Thue Gleichungen, Publ. Math. Debrecen 34 (1987), 189-196.

[20] A. Pethő and R. T. Tichy, On two-parametric quartic families of Diophantine problems, J. Symbolic Comput. 26 (1998), 151-171.

[21] N. P. Smart, The Algorithmic Resolution of Diophantine Equations, Cambridge Univ. Press, Cambridge, 1998.

[22] E. Thomas, Complete solutions to a family of cubic Diophantine equations, J. Number Theory 34 (1990), 235-250.

[23] A. Thue, Über Annäherungswerte algebraischer Zahlen, J. Reine Angew. Math. 135 (1909), 284-305.

[24] A. Togbé, On the solutions of a family of quartic Thue equations, Math. Comp. 69 (2000), 839-849.

[25] N. Tzanakis, Explicit solution of a class of quartic Thue equations, Acta Arith. 64 (1993), 271-283.

[26] N. Tzanakis and B. M. M. de Weger, On the practical solution of the Thue equation, J. Number Theory 31 (1989), 99-132.

[27] I. Wakabayashi, On a family of quartic Thue inequalities, ibid. 66 (1997), 70-84.

[28] —, On a family of quartic Thue inequalities, II, ibid. 80 (2000), 60-88.

Department of Mathematics

FESB

University of Zagreb

University of Split

Bijenička cesta 30

R. Boškovića bb

10000 Zagreb, Croatia 21000 Split, Croatia

E-mail:duje@math.hr

E-mail: borka@fesb.hr

Received on 15.1.2001

and in revised form on 23.3.2001 